\title{
A cohort study on safety for oocyte retrieval in IVF using the same 20G needle for local analgesia and retrieval.Vaginal cleaning did not add to the safety of the procedure
}

\begin{abstract}
Aim of study: What is the effectiveness and safety of using a $20 \mathrm{G}$ single lumen needle (SLN) for both applying local analgesia (LA) in the vaginal vault and ovarian capsule and oocyte retrieval (OR) using a simple syringe? And will thoroughly cleaning (TC) of the vagina before reducing the risk of the puncture procedure? Using a thin SLN has been postulated to jeopardize cumulus oocyte complexes (COC). Further applying LA in the same needle without retracting the needle before OR has been claimed to harm oocyte development. Also, many clinics extensively clean the vagina before OR.
\end{abstract}

Methods: We performed a retrospective cohort study including 4983 women. 877 underwent no cleaning (nonTC) of the vagina and 4106 underwent TC using $\mathrm{NaCl}$. All had OR with a $20 \mathrm{G}$ SLN attached to a syringe for local anaesthesia and egg collection. The treatments were done in one centre during the period from January 2016 to June 2019.

We studied women undergoing IVF treatment aged 18-45 years. Women had either thoroughly vaginal cleaning before OR or no cleaning depending on the physicians preferences. All women had LA using Citanest Dental Octapressin $2 \mathrm{ml}$. placed in the vaginal vault in the direction of the intended puncture including in the ovarian capsule. Therefore, only one puncture was needed in both sides, left and right. All punctures were hereafter followed by OR using the same $20 \mathrm{G}$ SLN. Aspiration was done by a $20 \mathrm{ml}$ syringe handled manually by the physician. All women underwent a conventional antagonist protocol with FSH stimulation and Ovitrelle 250IU for induction or Mild Stimulation using Tamoxifen, FSH and Ovitrelle. Outcome measurement were bleeding measured by the necessity of applying compression after the puncture or infection observed up to 1 month.

Results: Baseline characteristics including age, BMI and type of stimulation were comparable between the groups. In the nonTC group no infections, bleedings or abscesses were found. In the TC group there were 1 abscess observed. Intact COC was similar in the two groups as well as pregnancy rates.

Conclusion: During OR in ART the use of a 20G SLN using the same needle for LA and hereafter, without changing needle in the puncture channel, continue with the egg collection (EC) is simple and safe. In this situation TC is not necessary before puncture, making the procedure more patients friendly. In women undergoing oocyte retrieval for IVF, we found the use of a $20 \mathrm{G} \mathrm{SLN}$ for both applying LA and retrieving COC safe, effective and simple. Additional TC of the vagina before puncture did not add more to the safety of the procedure.

Keywords: IVF, art, egg collection, bleeding, infection, safety
Volume 12 Issue 4 - 202I

\author{
G] Almind, E Færch, F Lægaard, S Smidt- \\ Jensen, S Lindenberg \\ Copenhagen Fertility Center, Section for Reproduction, Lygten \\ 2c, 2400NV, Copenhagen Denmark
}

Correspondence: GJ Almind, Copenhagen Fertility Center Section for Reproduction. Lygten 2c, 2400NV, Copenhagen Denmark,Tel +453325 7000, Email gittejuul@gmail.com

Received: August 18,2021 | Published: August 27, 202

\section{Introduction}

Assisted reproductive technologies (ART) involve oocytes collection from the ovaries. The novel technology of using ultrasound guided transabdominal ${ }^{1}$ and later transvaginal ${ }^{2}$ was developed to make it safer and more patient friendly for the women undergoing ART compared to laparoscopic oocyte collection. However, some problems have been associated with this technology such as pain, bleeding and infection. ${ }^{3}$

The pain concurrent with the puncture was initially coped with by sedating or using general anaesthesia during the puncture. Even today several clinics are using this approach to comfort the patient. However, reducing the needle size from $15 \mathrm{G}$ to $17 \mathrm{G}$ initially reduced the problem with pain so we could perform the puncture using only sedatives like morphine and diazepam. Later the introduction of an even thinner needles and local analgesia even further reduced the need of sedation. Using the same thin needle without retracting the needle before OR has been claimed to harm oocyte development. ${ }^{4}$

The vaginal or intraabdominal bleeding and infection have been dealt with in several studies. ${ }^{3,5}$ From other disciplines of medicine the downsizing of the needle thickness always results in less pain, bleeding and infection risk. ${ }^{6}$

However, there is a limit to the smallest size of a puncture needle to be used for transabdominal and transvaginal puncture as a certain stiffness is needed for the procedure to manipulate the needle in the right position and also not to harm the COC. ${ }^{7}$ 
Further a discussion of the need to clean the vagina using chlorhexidine or $\mathrm{NaCl}$ before puncture has resolved in many procedures comprising washing of the vagina to avoid postoperative infection. This has now been challenged in other procedures such as vaginal surgery and induced abortion procedures. ${ }^{8,9}$

The aim of this study was to evaluate the safety and efficacy of using a single lumen $20 \mathrm{G}$ needle for LA and OR and without using thoroughly cleaning of the vagina as well before OR. Thoroughly cleaning is washing more than 30 seconds. The aim of this study was to make the OR procedure even safer and more patients friendly.

\section{Material and methods}

\section{Patient cohort and data collection}

We performed a retrospective cohort study at the Copenhagen Fertility Center in Denmark from January 2016 until June 2019. Women admitted to IVF/ICSI, were eligible for inclusion if they had one or more follicles for oocyte retrieval and had an Ovitrelle (Merck) human choriogonadotropin (hCG) injection 34-36 hours before the OR. Exclusion criteria were ongoing genital infection or other infections at the time of the OR.

\section{Hormone stimulation}

All women underwent either mild stimulation using Tamoxiphene 20-40mg daily and every second day 150IU FSH (Gonal F) until the follicular size were $17 \mathrm{~mm}$ and an injection of human choriongonadotropin (hCG) Ovitrelle (Merck) was given to induce final maturation. ${ }^{10}$

A short conventional antagonist protocol using FSH 100-250IU Gonal F daily depending on antral follicle count (AFC) and $0.25 \mathrm{mg}$ Orgalutran (SUN Pharma) was administrated daily to avoid premature ovulation. An injection of Ovitrelle was given to induce final maturation. Monitoring ultrasound scans were initiated on cycle day, CD3 and on CD9 in the cycle and on the day of ovulation induction.

\section{Oocyte retrieval procedure}

The patient was either washed in the vagina with several gauche swabs using isotonic saline or only inspected with a swab to exclude any foreign obstacles prior to puncture.

All punctures were performed using a Brüel and Kjær Flex Focus 500 ultrasound machine, and a dynamic vaginal probe $(7,5 \mathrm{MHz})$ with a disposable needle guide (Endocavity Needle Guide) attached. The needle (20G, $325 \mathrm{~mm}$ Kitazato single lumen OPU needle) was introduced trough the needle guide. When reaching the vaginal vault in close apposition to the ovary, Citanest in a total volume of up to two $\mathrm{ml}$ were applied in the vaginal wall and in the ovarian capsule using a two $\mathrm{ml}$ syringe attached to the needle. When the ovarian capsule was reached the Citanest syringe was exchanged with a $20 \mathrm{ml}$ syringe and the needle further introduced into the follicles. This procedure was then repeated on the contralateral site.

The syringe with the follicular fluid was delivered to the adjacent laboratory when the follicles where collected. If necessary, another syringe was applied to empty further follicles. In the laboratory the COC were identified and cultivated.

No other sedatives or intravenous administration of drugs were given during the procedure. The patient was kept under observation for 30 minutes in our observation room. Hereafter released from the clinic.

Insemination of mature oocytes were done either by regular IVF or ICSI 3-5 hours after OR and fertilisation check was performed 16
- 18 hours after insemination. Maturation check was done 68 hours after fertilization.

Embryo transfer was done day two or day three from OR depending on the former history or if wished so by the patient. All surplus embryos were cultured to blastocysts and cryopreserved if suitable. $95 \%$ of the ET where single embryo transfer. ET were not performed in the case of high risk for ovarian hyper stimulation syndrome (OHSS).

\section{Outcome measures}

The primary outcome was infection or bleeding observed in the period of 30 days after the puncture. Secondary outcomes were a fresh embryo transfer direct following the OR and clinical pregnancy. Clinical pregnancy was confirmed by ultrasound in the $7^{\text {th }}$ gestational week with a foetus in a gestational sac in the uterus.

\section{Statistical analysis}

The data were analysed using descriptive statistics. Differences between TC and nonTC groups were analysed using Student's t-test. $\mathrm{P}$-values $<0.05$ would be considered statistically significant.

\section{Results}

The mean age of the patients in both groups were 39 years. The numbers of intact $\mathrm{COC}$ were similar in the two groups as well as pregnancy rates. During the period all 4983 OC were included. A total of 11 different clinicians performed between 5 and 1037 OR. Two clinicians performed no cleaning of the vagina and nine did a thorough vaginal cleaning before OR. A total number of 877 patients had nonTC and 4106 patients had TC before OR. In the nonTC group no infections, bleedings or abscesses were found.

In the TC group there was observed one pelvic abscess. Straight after OPU intravenous Zinacef was administered because of endometriosis. Three months after OPU the patient came for a scan. Per oral antibiotics was initiated because of suspicion of infection. Ten days later the patient was admitted to the hospital for further treatment where the abscess was punctured and emptied. And in regard to the standard procedures in the hospital, she was treated with intravenous antibiotics. Several routine vaginal ultrasound scans were performed during the period.

In the TC group we found one patient that had a small vaginal bleeding right after OPU. Furthermore, a smaller intraabdominal bleeding was observed in the same patient. The vaginal bleeding was easily stopped with a mesch. No further treatment was necessary (Table 1).

Table I Women with standard conventional and mild stimulation

\begin{tabular}{lll}
\hline & No cleaning & Cleaning \\
\cline { 2 - 3 } & (non TC) & (TC group) \\
\hline Number of doctors & 2 & 9 \\
OR Cycles & 877 & 4106 \\
Mean age & 39 & 39 \\
Bleeding & 0 & $\mathrm{I}$ \\
Infections & 0 & $\mathrm{I}$ \\
Mean number of oocytes retrieved & 4.5 & 4.5 \\
Oocytes suitable for transfer on day 2-3 & 3.2 & 3.2 \\
\hline
\end{tabular}

In both groups $95 \%$ of all embryo transfers were single embryo transfer (SET). The mean number of oocytes retrieved in both groups 
was 4,5 and the number of embryos suitable for transfer on day 2 or 3 was 3,2 for both groups.

There were no significant differences between the two groups in cases of bleeding $(p=0.082)$, infection $(p=0.1766)$.

\section{Discussion}

This study demonstrates that it is feasible and simple to use a single lumen $20 \mathrm{G}$ needle first with local analgesics and direct hereafter, without retracting the needle, to perform the oocyte aspiration.

Intensive washing of the vagina before OR did not decrease the already known very low infection rate. Studies have shown that $\mathrm{NaCl}$ cleaning before abort and hysteroscopy do not reduce infection. From other studies it is already demonstrated that a $20 \mathrm{G}$ needle does not disrupt the COC although claimed previously. ${ }^{7}$ Even $23 \mathrm{G}$ needles can be used for this. However, when the needle gets thinner the rigidity is less and therefore the ability to penetrate tissue and aim at a specific follicle gets worse. By using a single lumen needle the diameter to pass for the follicle fluid and COC is larger than the same needle with a double lumen. In our case where flushing seldom is used, we used the single lumen needle to benefit from both a broader lumen and still be able to reduce the size of the needle diameter.

The reduction of the needle diameter has been shown in other studies to reduce infection and pain conception during puncture. ${ }^{6}$ In our study we can propose, that administrating only local analgesic infection and bleeding are not considerable. This was also shown by Kato. ${ }^{11}$

\section{Conclusion}

This study demonstrates that a single lumen needle $20 \mathrm{G}$ used both for applying a volume of local analgesic in the vaginal vault and the ovarian capsule, combined with no cleaning of the vagina is safe and effective, reducing the discomfort, pain and make the OR feasible without sedation.

\section{Acknowledgments}

None.

\section{Author contribution statement}

SL, GJA, EF, SSJ, FL did data collection and were all part of the clinical treatment of these patients. Including writing and correcting manuscripts. Moreover, FL did the statistical evaluation.

\section{Ethics statements}

\section{Studies involving animal subjects}

Generated Statement: No animal studies are presented in this manuscript.

\section{Studies involving human subjects}

Generated Statement: Ethical review and approval was not required for the study on human participants in accordance with the local legislation and institutional requirements. Written informed consent for participation was not required for this study in accordance with the national legislation and the institutional requirements.

\section{Inclusion of identifiable human data}

Generated Statement: No potentially identifiable human images or data are presented in this study.

\section{Funding}

None.

\section{Conflicts of interest}

The authors declare that the research was conducted in the absence of any commercial or financial relationships that could be construed as a potential conflict of interest.

\section{References}

1. Lenz S, Lauritsen JG, Kjellow M. Collection of human oocytes for in vitro fertilisation by ultrasonically guided follicular puncture. Lancet (London, England). 1981;1:1163-1164.

2. Wikland M, Nilsson L, Hansson R, et al. Collection of human oocytes by the use of sonography. Fertil Steril. 1983;39:603-608.

3. Medicine I, Council NR. Assessing the medical risks of human oocyte donation for stem cell research: workshop report. The National Academies Press; 2007.

4. Matsota P, Kaminioti E, Kostopanagiotou G. Anesthesia related toxic effects on in vitro fertilization outcome: burden of proof. Biomed Res Int. 2015.

5. El-Shawarby S, Margara R, Trew G, et al. A review of complications following transvaginal oocyte retrieval for in-vitro fertilization. Hum Fertil. 2004;7:127-133

6. Wago KJ, Skarsvåg TI, Lundbom JS, et al. The importance of needle gauge for pain during injection of lidocaine. J Plast Surg Hand Surg. 2016;50:115-118

7. Kyono K. P-898: Which is the best aspiration needle for ART? The 19, 20, or 21 gauge. Fertil Steril. 2006.

8. Kjolhede P, Halili S, Lofgren M. The influence of preoperative vaginal cleansing on postoperative infectious morbidity in abdominal total hysterectomy for benign indications. Acta Obstet Gynecol Scand. 2009;88:408-416.

9. Ludwig AK, Glawatz M, Griesinger G, et al. Perioperative and postoperative complications of transvaginal ultrasound-guided oocyte retrieval: prospective study of \&gt;1000 oocyte retrievals. Hum Reprod. 2006;21:3235-3240.

10. Almind G, Faerch E, Lindenberg F, et al. Mild stimulation approach for in vitro fertilization treatment: Retrospective data from one Danish Centre. Facts, views Vis. ObGyn. 2018;10:81-84

11. Kato K, Takehara Y, Segawa T, et al. Minimal ovarian stimulation combined with elective single embryo transfer policy: Age-specific results of a large, single-centre, Japanese cohort. Reprod Biol Endocrinol. 2012;10:1-7. 